\title{
The Relaxed Edge-Coloring Game and $k$-Degenerate Graphs
}

\author{
Charles Dunn \\ Linfield College \\ David Morawski \\ University of Minnesota \\ Jennifer Firkins Nordstrom \\ Linfield College
}

Follow this and additional works at: https://digitalcommons.linfield.edu/mathfac_pubs

Part of the Discrete Mathematics and Combinatorics Commons

\section{DigitalCommons@Linfield Citation}

Dunn, Charles; Morawski, David; and Nordstrom, Jennifer Firkins, "The Relaxed Edge-Coloring Game and k-Degenerate Graphs" (2015). Faculty Publications. Accepted Version. Submission 8.

https://digitalcommons.linfield.edu/mathfac_pubs/8

This Accepted Version is protected by copyright and/or related rights. It is brought to you for free via open access, courtesy of DigitalCommons@Linfield, with permission from the rights-holder(s). Your use of this Accepted Version must comply with the Terms of Use for material posted in DigitalCommons@Linfield, or with other stated terms (such as a Creative Commons license) indicated in the record and/or on the work itself. For more information, or if you have questions about permitted uses, please contact digitalcommons@linfield.edu. 


\title{
THE RELAXED EDGE-COLORING GAME AND $k$-DEGENERATE GRAPHS
}

\author{
CHARLES DUNN, DAVID MORAWSKI, AND JENNIFER FIRKINS NORDSTROM
}

\begin{abstract}
The $(r, d)$-relaxed edge-coloring game is a two-player game using $r$ colors played on the edge set of a graph $G$. We consider this game on forests and more generally, on $k$-degenerate graphs. If $F$ is a forest with $\Delta(F)=\Delta$, then the first player, Alice, has a winning strategy for this game with $r=\Delta-j$ and $d \geq 2 j+2$ for $0 \leq j \leq \Delta-1$. This both improves and generalizes the result for trees in [10]. More broadly, we generalize the main result in [10] by showing that if $G$ is $k$-degenerate with $\Delta(G)=\Delta$ and $j \in[\Delta+k-1]$, then there exists a function $h(k, j)$ such that Alice has a winning strategy for this game with $r=\Delta+k-j$ and $d \geq h(k, j)$.
\end{abstract}

\section{INTRODUCTION}

The map-coloring game was first conceived by Steven Brams and published by Martin Gardner in his column in Scientific American [17]. In this game two players alternate coloring the countries on a map such that no two countries with a non-trivial border receive the same color. It was not until 1991, that Bodlaender [2] reinvented it by framing the game in terms of coloring vertices of a graph.

In the original version of the game two players, Alice and Bob alternate coloring the vertices of a finite graph $G$ using colors from a set $X$ of $r$ colors. Alice goes first. On each turn, the players must choose an uncolored vertex to color such that at the end of the player's turn, no adjacent vertices have the same color. Alice wins this game if all of the vertices are eventually colored. Bob wins if there comes a time when there is an uncolored vertex for which no allowable color exists. The least $r$ such that Alice has a winning strategy for this game is called the game chromatic number of $G$, denoted $\chi_{\mathrm{g}}(G)$. This parameter has been investigated extensively in a number of papers $[8,16,18,20,21,25,26]$.

Observe that in the above game, at every step every color class induces an independent set in $G$, and that while playing the game, the players are in the process of creating a proper coloring of the graph. We first consider a variation of this game in which the players are creating a relaxed coloring,

Date: July 2013.

2000 Mathematics Subject Classification. 05C15, 05C57.

Key words and phrases. competitive coloring, $k$-degenerate graph, edge coloring.

Partially supported by the NSF grant DMS 0649068. 
also called defect coloring, of the the graph $G$. This variation was introduced by Chou et al. [4] and builds on the known work [5, 6, 7, 14, 24] concerning defect colorings of graphs. The only difference between this game and the original version is which colors the players are allowed to use. In this version of the game, a color $\alpha \in X$ is legal for an uncolored vertex $u$ if after $u$ is colored $\alpha$ each color class $H \subseteq G$ satisfies $\Delta(H) \leq d$, where $\Delta(H)$ is the maximum degree in $H$, and $d$ is a fixed nonnegative integer set at the beginning of the game. We call $d$ the defect. As in the original game, Alice wins if every vertex is eventually colored. Bob wins if there comes a time in the game when there is an uncolored vertex for which no legal color exists. We call this game the $(r, d)$-relaxed coloring game. For a fixed $d$, the least $r$ for which Alice has a winning strategy is called the $d$-relaxed game chromatic number of $G$, denoted ${ }^{d} \chi_{\mathrm{g}}(G)$. If $d=0$, we drop the initial superscript and write simply $\chi_{\mathrm{g}}(G)$. For a fixed $r$, the $r$-game defect of $G$, denoted $\operatorname{def}_{\mathrm{g}}(G, r)$, is the least $d$ such that Alice has a winning strategy. These parameters have been further examined in a number of papers, including $[11,12,13,9,19]$.

It should be noted that the game chromatic number has some interesting non-monotone properties. It is well known that $\chi_{\mathrm{g}}\left(K_{n, n}\right)=3$ for $n \geq 2$, but if $M$ is any perfect matching in $K_{n, n}$, then $\chi_{\mathrm{g}}\left(K_{n, n}-M\right)=n$. This gives an example of a graph $G$ with subgraph $H$ such that $\chi_{\mathrm{g}}(H)>\chi_{\mathrm{g}}(G)$. Extending this idea to the $(r, d)$-relaxed coloring game, it is well known that ${ }^{1} \chi_{\mathrm{g}}\left(K_{n, n}\right)=n$. This provides an example of a graph $G$ such that $\chi_{\mathrm{g}}(G)<$ ${ }^{1} \chi_{\mathrm{g}}(G)$. In fact, it was shown in [9] that for every $m \in \mathbb{N}$, there exists a graph $G$ such that $m \leq \chi_{\mathrm{g}}(G)<{ }^{1} \chi_{\mathrm{g}}(G)$. It remains open whether for all nonnegative integers $d$ there exists a graph $G$ such that ${ }^{d} \chi_{\mathrm{g}}(G)<{ }^{d+1} \chi_{\mathrm{g}}(G)$.

The focus of our work in this paper is a further variation of this game. Rather than coloring vertices, the players color edges. Of course, for a given graph $G$, this could be viewed as playing the $(r, d)$-relaxed coloring game game on the line graph of $G$. For the purposes of this paper, however, we will consider this game in terms of edge coloring. We call this variation the $(r, d)$-relaxed edge-coloring game and formalize it in the following way.

Let $G$ be a finite graph and let $r$ be a positive integer and $d$ be a nonnegative integer. As above, $d$ is the defect and $X$ is a set of $r$ colors. The players alternate coloring, with Alice coloring an edge first. We say that a color $\alpha \in X$ is legal for an uncolored edge $e$ if the following conditions are satisfied:

(1) The edge $e$ is incident with no more than $d$ edges already colored $\alpha$.

(2) If $e^{\prime}$ is an edge incident to $e$ and $e^{\prime}$ has already been colored $\alpha$, then $e^{\prime}$ is adjacent to no more than $d-1$ edges colored $\alpha$.

Note that if $e$ is colored $\alpha$, then at this point in the game, every edge has at most $d$ neighbors colored $\alpha$. Alice wins if every edge is eventually legally colored. Bob wins if there comes a time in the game when there is an uncolored edge for which no legal color exists. For a fixed defect $d$, the least 
$r$ such that Alice has a winning strategy for this game is called the $d$-relaxed game chromatic index of $G$, denoted ${ }^{d} \chi_{\mathrm{g}}^{\prime}(G)$. Similarly, for a fixed $r$, the $r$-edge-game defect of $G$, denoted $\operatorname{def}_{\mathrm{g}}^{\prime}(G, r)$, is the least $d$ such that Alice has a winning strategy. This game was first introduced in [10].

To provide the appropriate context for the work in this paper, we note that for the class of forests, much is already known about the $(r, d)$-relaxed (vertex) coloring game. We have the following sequence of results:

Theorem 1 (Faigle et al. [16]). If $F$ is a forest, then $\chi_{\mathrm{g}}(F) \leq 4$. Moreover, there exists a forest $F_{0}$ such that $\chi_{\mathrm{g}}\left(F_{0}\right)=4$.

Theorem 2 (Chou et al. [4]). If $F$ is a forest, then ${ }^{1} \chi_{\mathrm{g}}(F) \leq 3$. Moreover, there exists a forest $F_{1}$ such that ${ }^{1} \chi_{\mathrm{g}}\left(F_{1}\right)=3$.

Theorem 3 (He et al. [19]). If $F$ is a forest, then ${ }^{2} \chi_{\mathrm{g}}(F) \leq 2$. Moreover, there exists a forest $F_{2}$ such that ${ }^{2} \chi_{\mathrm{g}}\left(F_{2}\right)=2$.

From a certain perspective, this fully describes the $(r, d)$-relaxed coloring game on forests, as a class. The goal of this paper is to similarly describe the $(r, d)$-relaxed edge-coloring game on both forests and $k$-degenerate graphs. We begin by considering the following results:

Theorem 4 (Cai and Zhu [3], Lam et al. [23]). Let $T$ be a tree with $\Delta(T)=$ $\Delta$. Then $\chi_{\mathrm{g}}^{\prime}(T) \leq \Delta+2$.

Theorem 5 (Dunn [10]). Let $T$ be a tree with $\Delta(T)=\Delta$. Then $\operatorname{def}_{\mathrm{g}}^{\prime}(T, \Delta+$ $1) \leq 1$. Moreover, if $d \geq 1$, then ${ }^{d} \chi_{\mathrm{g}}^{\prime}(T) \leq \Delta+1$.

Theorem 6 (Dunn [10]). Let $T$ be a tree with $\Delta(T)=\Delta$. Then $\operatorname{def}_{\mathrm{g}}^{\prime}(T, \Delta) \leq$ 3. Moreover, if $d \geq 3$, then ${ }^{d} \chi_{\mathrm{g}}^{\prime}(T) \leq \Delta$.

We note that Theorems 4,5 and 6 can easily be extended to forests. Therefore, thinking in terms of Theorems 1-3, Theorems 4-6 lead to the following question:

Question 1. For any forest $F$ with $\Delta(F)=\Delta$ and $j \in[\Delta-1]$ is there a function $h: \mathbb{N} \rightarrow \mathbb{N}$ such that whenever $d \geq h(j)$ we have that ${ }^{d} \chi_{\mathrm{g}}^{\prime}(F) \leq$ $\Delta-j$ ?

In Section 2 of this paper we will answer Question 1 in the affirmative with $h(j)=2 j+2$. It should be noted that Theorem 4 was improved in [15]. It was shown that if $\Delta=3$ or $\Delta \geq 6$, then any tree $T$ with $\Delta(T)=\Delta$ satisfies $\chi_{\mathrm{g}}^{\prime}(T) \leq \Delta+1$. In [1], this result was extended to trees (and forests) with $\Delta=5$. The case for $\Delta=4$ remains open, indicating the difficulty of determining these parameters, even in the non-relaxed environment.

For the more general class of $k$-degenerate graphs, which will be defined in detail in Section 3, we note the following result of Cai and Zhu:

Theorem 7 (Cai and Zhu [3]). Let $G$ be a $k$-degenerate graph with $\Delta(G)=$ $\Delta$. Then $\chi_{\mathrm{g}}^{\prime}(G) \leq \Delta+3 k-1$. 
As a consequence, in terms of the $(r, d)$-relaxed edge-coloring game, we have that ${ }^{d} \chi_{\mathrm{g}}^{\prime}(G) \leq \Delta+3 k-1$ for all $k$-degenerate graphs $G$ when $d=0$. Also, in [10], the following result was proven:

Theorem 8 (Dunn [10]). Let $G$ be $k$-degenerate with $\Delta(G)=\Delta$. Then $\operatorname{def}_{\mathrm{g}}^{\prime}(G, \Delta+k-1) \leq 2 k^{2}+4 k$. Moreover, if $d \geq 2 k^{2}+4 k$, then ${ }^{d} \chi_{\mathrm{g}}^{\prime}(G) \leq$ $\Delta+k-1$.

Theorems 7 and 8 together lead to the following question:

Question 2. For any k-degenerate graph $G$ with $\Delta(G)=\Delta$ and $j \in[\Delta+$ $k-1]$ is there a function $h: \mathbb{N} \times \mathbb{N} \rightarrow \mathbb{N}$ such that whenever $d \geq h(k, j)$ we have that ${ }^{d} \chi_{\mathrm{g}}^{\prime}(G) \leq \Delta+k-j$ ?

In Section 3 we answer Question 2 in the affirmative. In particular, we will show that the following $h(k, j)$ suffices:

$$
h(k, j)= \begin{cases}2 k^{2}+4 k, & \text { if } j=1 ; \\ 2 k^{2}+4 k+2 j-4, & \text { if } j \geq 2 .\end{cases}
$$

We also derive corollaries for outerplanar graphs and planar graphs.

\section{The Relaxed Edge-Coloring Game on Forests}

Let $F=(V, E)$ be a forest with $\Delta(F)=\Delta$ for some positive integer $\Delta$. Let $T_{1}, T_{2}, \ldots, T_{n}$ be all components of $F$ containing at least one edge. For her strategy, for each $i \in[n]$ Alice chooses an arbitrary leaf $r_{i} \in V\left(T_{i}\right)$ at which she roots $T_{i}$. She then regards all edges in $T_{i}$ as oriented toward $r_{i}$. Let $E_{0}$ be the set of $n$ edges in $F$ that are incident to a root. For each vertex $v \in V \backslash\left\{r_{1}, r_{2}, \ldots, r_{n}\right\}$, define $p(v)$ to be the unique outneighbor of $v$. Then for each edge $e \in E$, there is a unique vertex $x \in V$ such that $e=x p(x)$. We now introduce some terminology, as illustrated in Figure 1.

For every edge $e=x p(x)$ with $p(x) \neq r_{i}$ for some $i \in[n]$, define the parent of $e$, denoted $p(e)$, to be the edge $p(x) p^{2}(x)$, where $p^{2}(x)=p(p(x))$. We say that $e$ is a child of $p(e)$. Note that, because $p(x)$ is well defined, $p(e)$ is also well defined. Whenever $p^{i}(e)$ is defined and $p^{i}(e)$ is not incident with the root, define $p^{i+1}(e)=p\left(p^{i}(e)\right)$. Define the descendants of $e$ to be

$$
G(e)=\left\{e^{\prime} \in E \mid e=p^{k}\left(e^{\prime}\right) \text { for some positive integer } k\right\} .
$$

For each edge $e=x p(x)$, define the siblings of $e$ to be

$$
B(e)=\{y p(y) \in E \mid p(y)=p(x) \text { and } y \neq x\}
$$

and $B[e]=B(e) \cup\{e\}$. Define the children of $e$ to be

$$
S(e)=\{y p(y) \in E \mid x=p(y)\} .
$$

We call the set of all edges incident to an edge $e$ the neighborhood of $e$, denoted $N(e)$.

Fix $j \in[\Delta-3]$ and let $X$ be a set of $\Delta-j$ colors. Note that $|X| \geq 3$. At any point in the game, let $C$ and $U$ be the set of colored and uncolored 


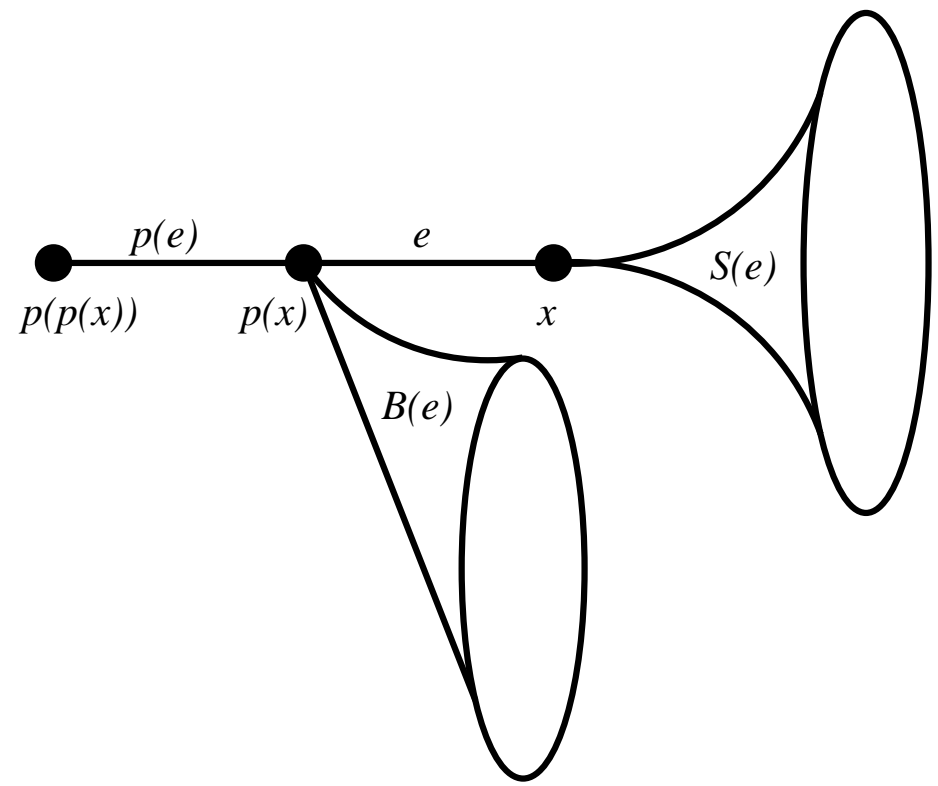

Figure 1. For an edge $e=x p(x)$, the vertex $p(p(x))=$ $p^{2}(x)$, the edge $p(e)$, and the sets $B(e)$ and $S(e)$.

edges, respectively. For each $\alpha \in X$, we call the set of all edges colored $\alpha$ the color class of $\alpha$, denoted $C_{\alpha}$. For a colored edge $e$, denote the color of $e$ by $c(e)$.

For each colored edge $e$, define the defect of $e$ to be the number of neighbors of $e$ colored with $c(e)$. If $e$ is uncolored, we set the defect of $e$ to be zero. We denote the defect of $e$ by $\operatorname{def}(e)$. Thus

$$
\operatorname{def}(e)= \begin{cases}\left|N(e) \cap C_{c(e)}\right|, & \text { if } e \in C \\ 0, & \text { otherwise }\end{cases}
$$

We say that color $\alpha \in X$ is eligible for edge $e$ if $p(e) \notin C_{\alpha}$. We denote the set of eligible colors for $e$ by $X(e)$. When coloring an edge $e$, Alice always chooses an eligible color. Note that since $|X| \geq 3$, this is always possible.

For any edge $e$, we say that $B[e]$ is secure if there exist edges $e_{1}, e_{2}, \ldots, e_{j+1} \in$ $B(e)$ and a color $\alpha$ such that $c\left(e_{i}\right)=\alpha$ for $i \in[j+1]$. In other words, $B[e]$ is secure if $e$ has $j+1$ siblings colored with the same color. Note that if $B[e]$ is secure, then the number of distinctly colored siblings of $e$ is at most

$$
\left|B(e) \backslash\left\{e_{1}, e_{2}, \ldots, e_{j}\right\}\right| \leq \Delta-j-2 .
$$

As $|X(e)| \geq \Delta-j-1$, there is always a legal eligible color for an uncolored edge $e$ when $B[e]$ is secure.

We will now define the strategy that Alice will use for this game with trees. This strategy is a modification of the activation strategy developed in [10]. In response to Bob's moves, Alice designates certain edges active; 
precisely how she chooses these edges will be explained below. When an edge $e$ becomes active, we say that $e$ has been activated. In addition, all colored edges are active. We denote the set of active edges by $A$, and remark that $C \subseteq A$. This set has the property that once an edge $e$ is in $A, e$ will remain active for the remainder of the game.

\section{Modified Forest Strategy}

Alice begins the game by coloring the unique edge in $E_{0}$ that is incident with $r_{1}$ with any color. Suppose now that Bob has just colored edge $b=x p(x)$ in $T_{i}$ for some $x \in V \backslash\left\{r_{1}, r_{2}, \ldots, r_{n}\right\}$ and some $i \in[n]$. Alice's response has two stages: a Search Stage and a Coloring Stage. In the Search Stage, Alice finds an edge $e$ to color. In the Coloring Stage, Alice chooses a color for $e$.

\section{Search Stage}

- If $b \in E_{0}$, then set $e$ to be any uncolored edge in $E_{0}$, if such an edge exists, or any uncolored edge whose parent is colored.

- If $p(b) \in U$, then activate each edge along the $\left(x, r_{i}\right)$-path until reaching either an edge $g$ with $g \in E_{0} \cap U$ or $p(g) \in A$. [Note that this includes Alice activating the edge b.] If $g \in E_{0} \cap U$ or $p(g) \in C$, set $e:=g$. Otherwise, set $e:=p(g)$.

- If $p(b) \in C$ with $c(p(b))=c(b)$ and $p^{2}(b) \in U$, then set $e:=p^{2}(b)$.

- If $p(b) \in C$ with $c(p(b))=c(b), p^{2}(b) \in C$, and $B(p(b)) \cap U \neq \emptyset$, then set $e$ to be any uncolored sibling of $p(b)$.

- Otherwise, set $e$ to be any uncolored edge in $E_{0}$, if such an edge exists, or any uncolored edge whose parent is colored.

\section{Coloring Stage}

- If $B[e]$ is secure, then color $e$ with an eligible color for $e$ that does not appear among the siblings of $e$.

- Otherwise, $B[e]$ is not secure. Let $f$ be the last edge to be colored with a color eligible for $e$ such that $c(f)=c(p(f))$ and $p(f) \in B(e)$. If such an edge exists, then color $e$ with $c(f)$. If no such edge exists, then color $e$ with any eligible color for $e$ that minimizes $\operatorname{def}(e)$.

We are now ready to state and prove our result for $(r, d)$-relaxed edgecoloring game on forests.

Theorem 9. Let $F=(V, E)$ be a forest and $\Delta(F)=\Delta$ for some positive integer $\Delta$. Let $j$ be an integer with $0 \leq j \leq \Delta-1$, and define $h(j)=2 j+2$. Then $\operatorname{def}_{\mathrm{g}}^{\prime}(F, \Delta-j) \leq h(j)$. Moreover, if $d \geq h(j)$ then ${ }^{d} \chi_{\mathrm{g}}^{\prime}(F) \leq \Delta-j$.

Proof. Suppose that Alice and Bob are playing the $(\Delta-j, d)$-relaxed edgecoloring game on $F$ for some $d \geq h(j)$. Note that when either $j=\Delta-1$ 
or $j=\Delta-2$, the result is immediate. Hence, it will suffice to consider the game with color set $X$ with $|X| \geq 3$. We will assume that Alice uses the Modified Forest Strategy.

Claim 1. If $e \in U$, then e has at most two active children. Furthermore, when e has two active children, Alice colors e.

Proof. Let $f$ be the first active child of $e$. When Alice activates $f$, she also activates $e$. Note that while $e$ is uncolored, Alice never colors an edge in $G(e) \backslash G(f)$ before Bob. If Bob colors an edge $b \in G(e) \backslash G(f)$, Alice activates $p(b), p^{2}(b), \ldots$, and so on, until she reaches $e$. Since $e$ is active, Alice colors $e$.

Claim 2. Suppose that Alice has chosen to color edge $e$ with $\alpha \in X$. Then at the end of Alice's turn, $\operatorname{def}(e) \leq j+2$.

Proof. Since $\alpha \in X(e)$, then $p(e)$ does not contribute to the defect of $e$. By Claim 1, $e$ has at most two active children; hence, $e$ has at most two children colored $\alpha$. If $B[e]$ is secure, then Alice would have chosen a color that does not appear among the siblings of $e$. In this case, $\operatorname{def}(e) \leq 2$. Otherwise, when $B[e]$ is not secure, there are at most $j$ siblings of $e$ colored $\alpha$. Thus, $\operatorname{def}(e) \leq j+2$.

Claim 3. Suppose that $e$ is about to be colored $\alpha$ and $p(e) \in U$. Then e has at most one child colored $\alpha$. Furthermore, if e has a child colored $\alpha$, then Bob colors e and Alice colors $p(e)$.

Proof. If some sibling $f \in B(e)$ is the first active child of $p(e)$, then Alice colors $p(e)$ when the first edge in $G(p(e)) \backslash G(f)$ is activated. Since $p(e)$ is uncolored and $e$ is to be colored, we conclude that $e$ has no active children and hence no children colored. So assume that $e$ is the first active child of $p(e)$. Note that $p(e) \in A$. If an edge in $G(p(e)) \backslash G(e)$ is then activated, Alice colors $p(e)$. Otherwise, we may assume that $e$ has no colored siblings at the time when $e$ is colored. Before $e$ is colored, it is incident with at most two colored edges, which are children of $e$. Since $|X| \geq 3$, there is a color that does not appear on any child of $e$. Then, because Alice will choose a color to minimize $\operatorname{def}(e)$, Alice never chooses to color $e$ with $\alpha$ if a child of $e$ has already been colored $\alpha$. So, if $e$ has two active children before $e$ is colored, then Alice colors $e$ with $\alpha$ only when neither child is colored $\alpha$. Thus, if $e$ has a child colored $\alpha$, then Bob must be coloring $e$ with $\alpha$, and since $p(e) \in A$, Alice responds by coloring $p(e)$.

Suppose $f \in S(e) \cap C_{\alpha}$. By Claim 3, if $f$ has a child colored $\alpha$ before $f$ is colored, then Bob must have colored $f$ and Alice responds by coloring $e$. Thus $\operatorname{def}(f)=2$ once $e$ is colored. Otherwise, $f$ has no children colored $\alpha$ before $f$ is colored. Since $e$ has at most two active children before $e$ is colored, $f$ has at most one sibling colored $\alpha$ before $e$ is colored. Then $\operatorname{def}(f) \leq 2$ once $e$ is colored. 
Now consider the siblings of $e$. If $B[e]$ is secure, since Alice is choosing to color $e$ with $\alpha$, then $\alpha$ does not appear among the siblings of $e$. Hence, coloring $e$ with $\alpha$ does not affect the defect of any edge in $B(e)$.

Finally, we consider the case that $B[e]$ is not secure.

Claim 4. Suppose Alice has chosen to color edge $e$ with $\alpha \in X$ and $B[e]$ is not secure. If there exists an edge $f \in B(e) \cap C_{\alpha}$, then $\operatorname{def}(f) \leq 2 j+2$ once $e$ is colored.

Proof. Let $E^{\prime}=B(e) \cap C_{\alpha}$. Since $B[e]$ is not secure, we have that $\left|E^{\prime}\right| \leq j$. Let $f \in E^{\prime}$ such that $\left|S(f) \cap C_{\alpha}\right|$ is maximal, and let

$$
S(f) \cap C_{\alpha}=\left\{s_{1}, s_{2}, \ldots, s_{m}\right\},
$$

where $i<j$ implies that $s_{i}$ is colored before $s_{j}$. We show that $m \leq\left|E^{\prime}\right|+2$. By Claim 1, $f$ has at most two active children before it is colored. Hence, $f$ has at most two children colored $\alpha$ before $f$ is colored. So only the following cases need be considered:

Case 1: The edge $f$ is colored before $s_{1}$.

Since $p\left(s_{i}\right)=f$ for each $i \in[n]$ and $c(f)=\alpha$, Alice does not color any $s_{i}$. For each $s_{i}$ that Bob colors, Alice then colors $p(e)$ if $p(e) \in U$, an edge in $E^{\prime} \backslash\{f\}$ if $p(e) \in C$, or $e$ if $E^{\prime} \cap U=\emptyset$. Then at most $\left|E^{\prime}\right|+1$ children of $f$ are colored $\alpha$ before Alice colors $e$. Hence, $m \leq\left|E^{\prime}\right|+1$.

Case 2: The edge $f$ is colored after $s_{1}$ and before $s_{2}$.

Alice does not color $s_{i}$ for any $i \geq 2$. As in the previous case, when Bob colors $s_{i}$ with $i \geq 2$, Alice then colors $p(e)$, an edge in $E^{\prime} \backslash\{f\}$, or $e$. Thus, once $f \in C$, at most $\left|E^{\prime}\right|+1$ children of $f$ are colored $\alpha$. Including $s_{1}$, we have that $m \leq\left|E^{\prime}\right|+2$.

Case 3: The edge $f$ is colored after $s_{2}$.

If $p(e) \in U$, then Claim 3 implies that $f$ has at most one child colored $\alpha$ before $f$ is colored. Since $f$ has two children colored $\alpha$ before $f$ is colored, $p(e)$ must be colored before $f$. Furthermore, Alice colors $f$ immediately after $s_{2}$ is colored, as $s_{1}$ and $s_{2}$ must be the first two active children of $f$. Once $f$ is colored, each time Bob colors a child of $f$ with $\alpha$, Alice colors an edge in $E^{\prime} \backslash\{f\}$. Therefore, once $f$ is colored, Bob can color at most $\left|E^{\prime}\right|$ children of $f$ with $\alpha$ before $e$ is colored. So $m \leq\left|E^{\prime}\right|+2$.

Thus we have that

$$
m=\left|S(f) \cap C_{\alpha}\right| \leq\left|E^{\prime}\right|+2 \leq j+2
$$

once $e$ is colored. Since $f$ was chosen to maximize $\left|S(f) \cap C_{\alpha}\right|$ and each $f^{\prime} \in E^{\prime}$ has at most $j$ siblings colored $\alpha$ before $e$ is colored, we have that

$$
\operatorname{def}\left(f^{\prime}\right) \leq\left|S(f) \cap C_{\alpha}\right|+j \leq 2 j+2
$$

for all $f^{\prime} \in E^{\prime}$. 
Note now that if Alice is coloring edge $e$ with $\alpha$, according to the Modified Forest Strategy, Claim 2 guarantees that $\operatorname{def}(e) \leq h(j)$. We have also shown that for any edge $f \in N(e) \cap C_{\alpha}$, immediately after $e$ is colored, $\operatorname{def}(f) \leq$ $h(j)$. As Bob may adopt Alice's strategy at any point in the game, every edge is eventually colored, and Alice wins the game. Thus

$$
\operatorname{def}_{\mathrm{g}}^{\prime}(F, \Delta-j) \leq 2 j+2=h(j) .
$$

Moreover, if the game is being playing with some defect $d>2 j+2$, and an edge $e$ eventually has defect at least $d$, then it must be through the actions of Bob that this occurs. At the time that $e$ is uncolored, the above arguments show that it is possible to color $e$ with an eligible color $\alpha$ such that coloring $e$ does not increase the defect of any edge $e^{\prime}$ with $\operatorname{def}\left(e^{\prime}\right)>2 j+2$. Thus, for any $d \geq h(j)$, we have that

$$
{ }^{d} \chi_{\mathrm{g}}^{\prime}(F) \leq \Delta-j,
$$

as desired.

We note that the above theorem generalizes the bound in Theorem 6 (Theorem 3 in [10]). Moreover, for $j=0$, Theorem 9 provides that for a forest $F$ with $\Delta(F)=\Delta$, we have that $\operatorname{def}_{\mathrm{g}}^{\prime}(F, \Delta) \leq 2$. This is an improvement by 1 of Theorem 6 .

\section{The Relaxed Edge-Coloring Game on $k$-Degenerate Graphs}

As discussed in Section 1, we now consider the $(r, d)$-relaxed edge-coloring game on the class of $k$-degenerate graphs. Recall that a graph $G=(V, E)$ is $k$-degenerate if there exists a linear ordering $L=v_{1}, v_{2}, \ldots, v_{n}$ of $V$ such that for every $i \in[n]$, we have that

$$
\mid\left\{j \mid v_{i} \leftrightarrow v_{j} \text { and } j<i\right\} \mid \leq k .
$$

For example, trees and forests are 1-degenerate, outerplanar graphs are 2 -degenerate, planar graphs are 5-degenerate, and partial $k$-trees are $k$ degenerate.

We now develop some necessary notation and terminology for our strategy with $k$-degenerate graphs. Let $G=(V, E)$ be a finite graph and let $L$ be a linear ordering of $V$. Once $L$ is established, when we write $x y \in E$ we assume that $x<y$ in $L$. We will also assume that the edge $x y$ is oriented $y \rightarrow x$. Let $e=x y$ be an edge in $G$. As in [10], we now define sets of edges relative to $e$ using $L$ and this orientation of $E(G)$.

$$
\begin{array}{rlrl}
P(e) & =\left\{w x \in E \mid w \in N^{+}(x)\right\} & & P[e]=P(e) \cup\{e\} \\
B(e) & =\left\{x v \in E \mid v \in N^{-}(x)\right\} & & B[e]=B(e) \cup\{e\} \\
H(e) & =\left\{u y \in E \mid u \in N^{+}(y)\right\} & H[e]=H(e) \cup\{e\} \\
S(e) & =\left\{y z \in E \mid z \in N^{-}(y)\right\} & & S[e]=S(e) \cup\{e\} \\
R(e) & =P(e) \cup H(e) & &
\end{array}
$$




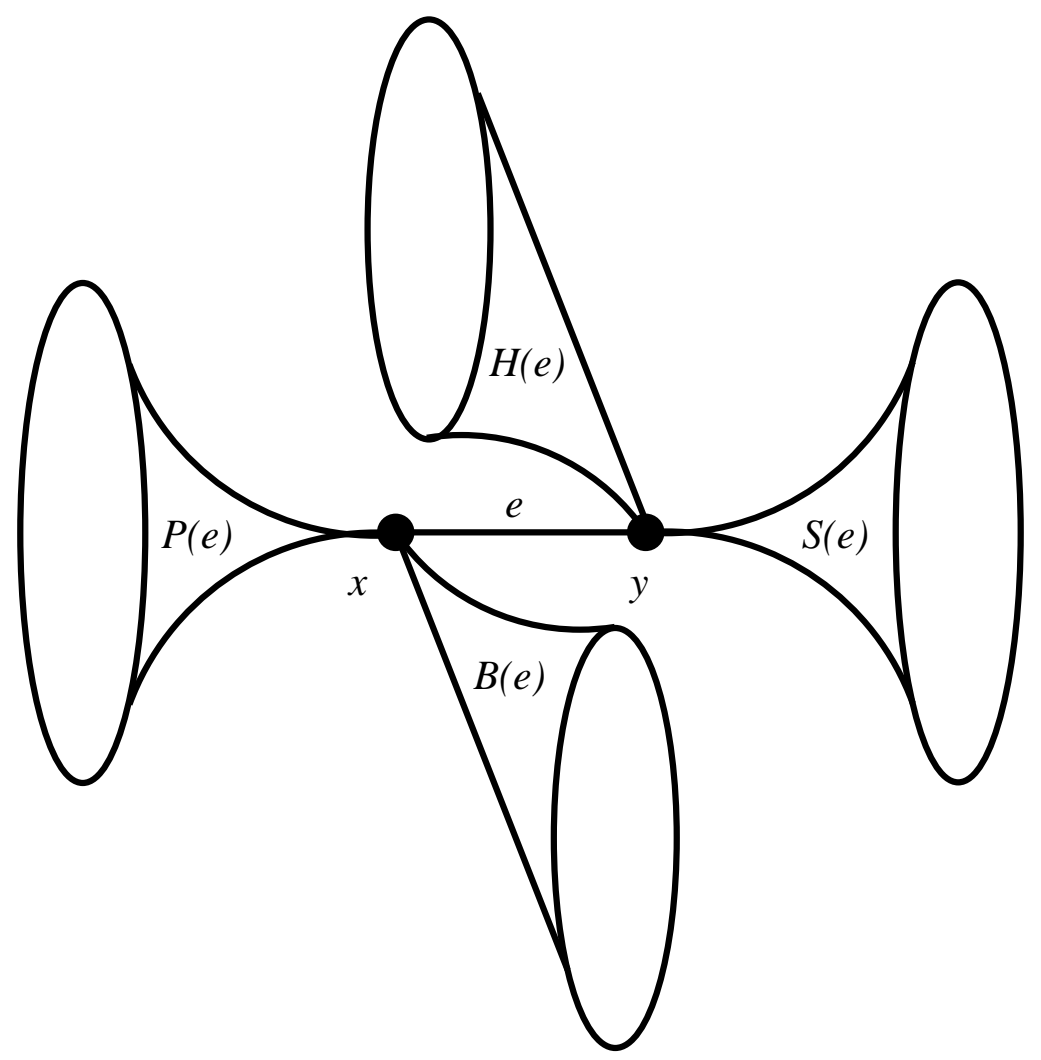

FiguRE 2. For an edge $e$ and linear ordering $L$, sets $P(e)$, $H(e), B(e)$, and $S(e)$, relative to $e$ and $L$.

We call the edges in $P(e)$ the parents of $e$ and the edges in $S(e)$ the children of $e$. We note that $N(e)=R(e) \cup S(e) \cup B(e)$. See Figure 2 .

Consider the linear ordering $\bar{L}$ of $E$ induced lexicographically from $L$. So $x y<w z$ in $\bar{L}$ if and only if either $x<w$ in $L$ or both $x=w$ and $y<z$ in $L$. Although $\bar{L}$ is used in the strategy below, since $\bar{L}$ is determined by $L$, it is $L$ that determines the strategy.

As with the game on trees, at any time in the game we define $U$ to be the set of uncolored edges and $C$ to be the set of colored edges. For $e \in C$, let the color assigned to $e$ be $c(e)$. Define the defect set of $e$ by $D(e)=\left\{e^{\prime} \in N(e) \mid c\left(e^{\prime}\right)=c(e)\right\}$. If $e \in U$, then $D(e)=\emptyset$. In either case, the defect of $e$ is defined by $\operatorname{def}(e)=|D(e)|$.

Let $F \subseteq E$. We define

$$
\bar{X}(F)=\bigcup_{e^{\prime} \in F}\left\{c\left(e^{\prime}\right)\right\}
$$


THE RELAXED EDGE-COLORING GAME AND $k$-DEGENERATE GRAPHS

where $\left\{c\left(e^{\prime}\right)\right\}=\emptyset$ if $e^{\prime} \in U$. For an edge $e$, we then let $X(e)=X-\bar{X}(R(e))$. In other words, $X(e)$ is the set of colors not used on any edge in $R(e)$. As before, we call $X(e)$ the set of eligible colors for $e$.

Let $e \in E$. We define $M(e)=U \cap P[e]$. If $M(e) \neq \emptyset$, we define the mother of $e$ by $m(e)=\min _{\bar{L}} M(e)$. So the mother of $e$, if it exists, is either the $\bar{L}$-least uncolored parent of $e$, or $e$ itself. Note that if $e \in U$ then $m(e)$ must be defined since $e$ is a candidate. For any colored edge $e$ we define $D^{*}(e) \subseteq D(e)$ by

$$
D^{*}(e)=\left\{e^{\prime} \in D(e) \mid m\left(e^{\prime}\right) \text { exists }\right\} .
$$

Using this definition, for a colored edge $e$, let $F(e)=P(e) \cap\left(U \cup D^{*}(e)\right)$. If $F(e) \neq \emptyset$, define the father of $e$ by $f(e)=\min _{\bar{L}} F(e)$. So the father of $e$, if it exists, is either the $\bar{L}$-least uncolored parent of $e$, or the $\bar{L}$-least parent of $e$ colored $c(e)$ whose mother exists.

We now assume that $G$ is $k$-degenerate and that $L$ is a linear ordering witnessing this. Let $\Delta(G)=\Delta$. Alice and Bob will be playing the $(r, d)$ relaxed edge-coloring game with $\Delta+k-j$ colors, for some $j \in[\Delta+k-1]$. Extending the idea with forests above, we define $B[e]$ to be secure if there exist edges $e_{1}, e_{2}, \ldots, e_{j+k-1} \in B(e)$ and a color $\alpha$ such that $c\left(e_{i}\right)=\alpha$ for $i \in[j+k-1]$. Finally, we define $l(e)=\min _{\bar{L}} B(e) \cap U$, if such an edge exists.

We are now ready to define the strategy that Alice will employ in the $(r, d)$-relaxed edge-coloring game on $k$-degenerate graphs. This is a modification and extension of the strategy developed in [10].

\section{Modified $K$ Strategy}

Let $G=(V, E)$ be a $k$-degenerate graph with a linear ordering $L$ of $V$ witnessing this. Let $X$ be a set of colors. Alice will again maintain an edge set $A$ of active edges. Similar to the case with forests, we note that all colored edges are active. Alice starts by activating and coloring the least edge in $\bar{L}$. Suppose that Bob has just colored edge $b$. First Alice activates $b$. Then, as in the Modified Forest Strategy, Alice's response has two stages: a Search Stage and a Coloring Stage. In the Search Stage, Alice finds an edge $e$ to color. The Search Stage includes an Initial Step and a Recursive Step. In the Coloring Stage, Alice chooses a color for $e$.

\section{Search Stage}

Initial Step

- If $f(b)$ exists and $f(b) \in U$, then set $g:=f(b)$ and move to the Recursive Step.

- If $f(b)$ exists and $f(b) \in C$, then set $g:=m(f(b))$ and move to the Recursive Step.

- If $f(b)$ does not exists and $l(b)$ exists, then set $e:=l(b)$ and move to the Coloring Stage. 
- Otherwise, set $e:=\min _{\bar{L}} U$. If $e$ is inactive, activate it. Move to the Coloring Stage.

Recursive Step

- If $g \notin A$, then activate $g$, set $g:=m(g)$, and repeat the Recursive Step.

- Otherwise, set $e:=g$ and move to the Coloring Stage.

\section{Coloring Stage}

- If $B[e]$ is secure, then color $e$ with an eligible color for $e$ that does not appear in $B(e)$.

- Otherwise, $B[e]$ is not secure. Let $f$ be the last edge to be colored with a color eligible for $e$ such that $c(f)=c\left(f^{\prime}\right)$ for some $f^{\prime} \in B(e) \cap P(f)$. If such an edge exists, then color $e$ with $c(f)$. If no such edge exists, then color $e$ with any eligible color for $e$ that minimizes $\operatorname{def}(e)$.

Note that three of the options for response in the Initial Step are to move from $b$ to $f(b), m(f(b))$, and $l(b)$. We will refer to these actions as jumping, skipping, and sliding, respectively. When Alice either activates or colors an edge $e$, we say that she is taking action at $e$. Note that Alice can take action at a edge at most twice. As before, this will play an important role in the proof of our main result, which we are now ready to state and prove.

Theorem 10. Let $G$ be $k$-degenerate with $\Delta(G)=\Delta$. Let $j \in[\Delta+k-1]$. Let

$$
h(k, j)= \begin{cases}2 k^{2}+4 k, & \text { if } j=1 ; \\ 2 k^{2}+4 k+2 j-4, & \text { if } j \geq 2 .\end{cases}
$$

Then $\operatorname{def}_{\mathrm{g}}^{\prime}(G, \Delta+k-j) \leq h(k, j)$. Moreover, if $d \geq h(k, j)$ then ${ }^{d} \chi_{\mathrm{g}}^{\prime}(G) \leq$ $\Delta+k-j$.

Proof. We begin by noting that if $\Delta-k \leq j \leq \Delta+k-1$ and $G$ has at least two edges, then

$$
\begin{aligned}
h(k, j) & =2 k^{2}+4 k+2 j-2 \\
& \geq 2 k^{2}+4 k+(2 \Delta-2 k)-2 \\
& =2 \Delta-2+2 k^{2}+2 k \\
& >2 \Delta-2 .
\end{aligned}
$$

However, an edge $e$ in $G$ is incident with at most $2 \Delta-2$ other edges. Since our allowable defect is greater than this, Alice can always win. Also, if $G$ has only one edge, the result is trivial. So for the remainder of our argument, we will assume that $j \in[\Delta-k-1]$. Let $X$ be a set of colors with $|X|=\Delta+k-j$. We will assume that Alice uses the Modified $K$ Strategy. We first show throughout the game, if $e \in U$, then $e$ has at least one legal eligible color. Note that $|P(e)| \leq k$ and $|H(e)| \leq k-1$. First suppose that 
$B[e]$ is not secure. Thus, the only colors that Alice must avoid are those used on edges in $R(e)$. So we have that

$$
\begin{aligned}
|X(e)| & =|X-\bar{X}(R(e))| \\
& \geq \Delta+k-j-|R(e)| \\
& =\Delta+k-j-|P(e) \cup H(e)| \\
& \geq \Delta+k-j-(2 k-1) \\
& =\Delta-k-j+1 \\
& \geq 1 .
\end{aligned}
$$

If $B[e]$ is secure, then we must show that the number of distinctly colored edges in $R(e) \cup B(e)$ is less than $\Delta+k-j$. Let $m$ be this number. First note that since $B[e]$ is secure, then within $B(e)$, at least $k+j-1$ edges are colored the same. So

$$
\begin{aligned}
m & \leq|R(e) \cup B(e)|-(k+j-2) \\
& =|P(e) \cup B(e)|+|H(e)|-(k+j-2) \\
& \leq \Delta-1+(k-1)-(k+j-2) \\
& =\Delta-j \\
& <\Delta+k-j .
\end{aligned}
$$

Thus, every uncolored edge has a legal eligible color. Suppose Alice is about to color edge $e$ with $\alpha \in X$. We consider the defect of both $e$ and any $\alpha$-colored neighbors of $e$. We will show that immediately after Alice colors $e$, none of these edges has defect greater than $h(k, j)$.

Claim 1. If $e \in U$, then e has at most $2 k$ children colored $\alpha$.

Proof. Let $e^{\prime} \in S(e) \cap C_{\alpha}$. Note that $\alpha \in X(e)$ implies that $\alpha$ is not used on any edge in $H(e)$. When $e^{\prime}$ is activated or colored, Alice does not skip, as this would require that both $e^{\prime}$ and $f\left(e^{\prime}\right) \in H[e]$ are colored $\alpha$. Also, Alice does not slide, since $e$ is a candidate for $f(e)$. Thus, Alice takes action in $H[e]$. So we have that

$$
\left|S(e) \cap C_{\alpha}\right| \leq 2|H[e]| \leq 2 k .
$$

Claim 2. If $e \in U$, then $e$ is incident with at most $3 k+j-2$ edges colored $\alpha$.

Proof. Since $\alpha \in X(e)$, then $\left|R(e) \cap C_{\alpha}\right|=0$. So it will suffice to show

$$
\left|S(e) \cap C_{\alpha}\right|+\left|B(e) \cap C_{\alpha}\right| \leq 3 k+j-2 .
$$

By Claim 1, we have that

$$
\left|S(e) \cap C_{\alpha}\right| \leq 2 k
$$


Now, if $B[e]$ is secure, then $\left|B(e) \cap C_{\alpha}\right|=0$. Otherwise, there are at most $j+k-2$ edges in $B(e)$ colored $\alpha$. Thus, in either case,

$$
\left|B(e) \cap C_{\alpha}\right| \leq j+k-2 .
$$

Hence,

as desired.

$$
\left|S(e) \cap C_{\alpha}\right|+\left|B(e) \cap C_{\alpha}\right| \leq 3 k+j-2,
$$

By Claim 2, immediately after Alice colors $e$ with $\alpha$, we have that $\operatorname{def}(e) \leq$ $3 k+j-2<h(k, j)$. We will now consider the defect of a child of $e$ already colored $\alpha$.

Claim 3. If $g \in S(e) \cap C_{\alpha}$, then before e is colored $\operatorname{def}(g) \leq 2 k^{2}+4 k-1$.

Proof. Observe that since $B(g) \subset S(e)$ and $g \notin D(g)$, Claim 1 implies that $|D(g) \cap B(g)| \leq 2 k-1$. We also have that $P(g)=H[e]$, implying that $P(g) \cap D(g)=\emptyset$. Let $|D(g) \cap H(g)|=a$, noting that $0 \leq a \leq k-1$, by our choice of $L$. Thus we have that

$$
\begin{aligned}
|D(g)| & =|D(g) \cap P(g)|+|D(g) \cap B(g)|+|D(g) \cap H(g)|+|D(g) \cap S(g)| \\
& \leq 2 k-1+a+|D(g) \cap S(g)| .
\end{aligned}
$$

Thus, it will suffice to show that $|D(g) \cap S(g)| \leq 2 k^{2}+2 k-a$.

Let $S=D(g) \cap S(g)$. We partition $S$ into $\left\{S_{1}, S_{2}\right\}$. We define $S_{1}$ to be the set of edges $e^{\prime}$ where Alice responds to the activation of $e^{\prime}$ by jumping and, therefore, taking action at an edge in $H[g]$. Similarly, $S_{2}$ is the set of edges $e^{\prime}$ where Alice responds to the activation of $e^{\prime}$ by skipping. Note that Alice will not respond by sliding since $e$ is uncolored. At first, we have that $\left|S_{1}\right| \leq 2 k$. However, this would imply that Alice both activates and colors the edges in $H[g]$. But she can color at most one edge in $H[g]$ with $\alpha$. Since

$$
\left|C_{\alpha} \cap H(g)\right|=|D(g) \cap H(g)|=a,
$$

we have that $\left|S_{1}\right| \leq 2 k-a$.

Now let $e^{\prime} \in S_{2}$. Let $Q=\bigcup_{h \in H[g]} P(h)$. Since Alice will skip once $e^{\prime}$ is activated, she next will take action at an edge in $Q$. Therefore, as Alice can take action at most twice at any given edge, we have that $\left|S_{2}\right| \leq 2|Q| \leq 2 k^{2}$. Hence, we have

$$
|S|=\left|S_{1}\right|+\left|S_{2}\right| \leq 2 k^{2}+2 k-a,
$$

as desired.

So by Claim 3 once $e$ is colored, $\operatorname{def}(g) \leq 2 k^{2}+4 k$ for any child of $e$ already colored $\alpha$. If $j=1$, then $\operatorname{def}(g) \leq h(k, j)$, since $h(k, 1)=2 k^{2}+4 k$. Otherwise, if $j \geq 2$, we note that

$$
h(k, j)=2 k^{2}+4 k+2 j-4 \geq 2 k^{2}+4 k .
$$

Thus, in either case, $\operatorname{def}(g) \leq h(k, j)$.

Finally, we consider the effect of coloring $e$ with $\alpha$ on any sibling of $e$ already colored $\alpha$. 
Claim 4. If $g \in B(e) \cap C_{\alpha}$, then before $e$ is colored $\operatorname{def}(g) \leq 2 k^{2}+4 k+2 j-5$.

Proof. Note that if $B[e]$ is secure, then $B(e) \cap C_{\alpha}=\emptyset$, as Alice will not choose to color $e$ with any color already used in $B(e)$. Thus, we may assume that $B[e]$ is not secure. As in Claim 3 , it will suffice to consider

$$
\begin{aligned}
\operatorname{def}(g) & =|D(g)| \\
& =|D(g) \cap P(g)|+|D(g) \cap B(g)|+|D(g) \cap H(g)|+|D(g) \cap S(g)| .
\end{aligned}
$$

Since $P(g)=P(e)$, we have that $|D(g) \cap P(g)|=0$. Let $|D(g) \cap H(g)|=a$, noting that $0 \leq a \leq k-1$, by our choice of $L$. Also, since $B[e]$ is not secure, then $|D(g) \cap B(g)| \leq j+k-3$. It then suffices to show that

$$
|D(g) \cap S(g)| \leq 2 k^{2}+3 k+j-2-a .
$$

Let $S=D(g) \cap S(g)$, and partition $S$ into $\left\{S_{1}, S_{2}, S_{3}\right\}$. As in Claim 3, we define $S_{1}$ to be the set of edges $e^{\prime}$ which Alice responds to the activation of $e^{\prime}$ by jumping and, therefore, taking action at an edge in $H[g]$. Similarly, $S_{2}$ is the set of edges $e^{\prime}$ which Alice responds to the activation of $e^{\prime}$ by skipping. Finally, $S_{3}$ is the set of edges $e^{\prime}$ which Alice responds to the activation of $e^{\prime}$ by sliding. As in our argument in Claim $3,\left|S_{1}\right| \leq 2 k-a$ and $\left|S_{2}\right| \leq 2 k^{2}$.

Let $e^{\prime} \in S_{3}$. Since Alice responds by sliding, she colors an edge in $B(g)$ with $\alpha$. Including the possible move on which she colors $e$, we have that $\left|S_{3}\right| \leq k+j-2$. Hence

$$
|S| \leq 2 k-a+2 k^{2}+k+j-2=2 k^{2}+3 k+j-2-a,
$$

as desired.

Thus, by Claim 4, once $e$ is colored $\alpha$, we have that

$$
\operatorname{def}(g) \leq 2 k^{2}+4 k+2 j-4
$$

for any sibling of $e$ already colored $\alpha$. Note that if $j=1$, then $h(k, j)=$ $2 k^{2}+4 k$, and $\operatorname{def}(g) \leq h(k, j)$. Otherwise, if $j \geq 2$, we have $h(k, j)=$ $2 k^{2}+4 k+2 j-4$, and again we have that $\operatorname{def}(g) \leq h(k, j)$.

We make two final observations. First, Bob can always borrow this strategy to find a legal move. Second, if $d>h(k, j)$ and an edge $e$ eventually has defect at least $d$ then it must be through the actions of Bob that this has occurred. At the time an edge $e$ is uncolored, the above arguments show that it is possible to color $e$ with an eligible color $\alpha$ such that coloring $e$ did not increase the defect of any edge $e^{\prime}$ where $\operatorname{def}\left(e^{\prime}\right)>h(k, j)$. Thus, every edge will eventually be colored, and Alice will win the game.

We note that Theorem 10 generalizes Theorem 8 (Theorem 5 in [10]). Additionally, while Theorem 10 includes forests when $k=1$, Theorem 9 provides a better bound. Hence, we suspect strongly that there may be room for improvement in the bound given by $h(k, j)$ in Theorem 10 .

Using the fact that outerplanar graphs are 2-degenerate and planar graphs are 5-degenerate, we have the following corollaries to Theorem 10: 
Corollary 11. Let $G$ be an outerplanar graph with $\Delta(G)=\Delta$ and let $j \in[\Delta+1]$. Let

$$
h(j)= \begin{cases}16, & \text { if } j=1 ; \\ 2 j+12, & \text { if } j \geq 2 .\end{cases}
$$

Then $\operatorname{def}_{\mathrm{g}}^{\prime}(G, \Delta+2-j) \leq h(j)$. Moreover, if $d \geq h(j)$ then ${ }^{d} \chi_{\mathrm{g}}^{\prime}(G) \leq$ $\Delta+2-j$.

Corollary 12. Let $G$ be a planar graph with $\Delta(G)=\Delta$ and let $j \in[\Delta+4]$. Let

$$
h(j)= \begin{cases}70, & \text { if } j=1 ; \\ 2 j+66, & \text { if } j \geq 2 .\end{cases}
$$

Then $\operatorname{def}_{\mathrm{g}}^{\prime}(G, \Delta+5-j) \leq h(j)$. Moreover, if $d \geq h(j)$ then ${ }^{d} \chi_{\mathrm{g}}^{\prime}(G) \leq$ $\Delta+5-j$.

Observe that taken together, Corollaries 11 and 12 generalize, and in fact include as a corollary, Corollary 1 in [10].

\section{Future Work}

Thinking in terms of Theorem 4 and the work in [15], it is evident that even the game chromatic index is difficult to determine for trees and forests. Thus, determining the tightness of the bounds in Theorems 5, 6, and 9 for the defect remain open problems. Additionally, the fact that the bound for the defect in Theorem 10 is not as good for trees and forests as in Theorem 9 seems to imply that the results in Theorem 10 have room for improvement. Our goal in this paper was first to show that such a bound exists. Future work will ideally lead to tighter bounds.

Finally, there are many properties of $\chi_{\mathrm{g}}^{\prime}(G)$ and ${ }^{d} \chi_{\mathrm{g}}^{\prime}(G)$ that remain to be studied. As discussed in the Introduction, both $\chi_{\mathrm{g}}(G)$ and ${ }^{d} \chi_{\mathrm{g}}(G)$ have somewhat unexpected non-monotone properties, both in terms of subgraphs and increasing the defect. It remains open to determine whether this is also the case with edge coloring. We suspect the answer is yes, but have yet to determine appropriate examples. Related to this question, it would be interesting to determine whether the difference between $\chi_{\mathrm{g}}^{\prime}(G)$ and ${ }^{d} \chi_{\mathrm{g}}^{\prime}(G)$ can be bounded by a function of $d$, or if ${ }^{d} \chi_{\mathrm{g}}^{\prime}(G)$ can be bounded by other graph parameters. While some of these questions have been explored for $\chi_{\mathrm{g}}(G)$, very little, if any, has been studied for the relaxed version of the games, either with vertex coloring or edge coloring.

\section{REFERENCES}

[1] S.D. Andres, "The game chromatic index of forests of maximum degree $\Delta \geq 5$," Discrete Applied Mathematics, 154 (2006), no. 9, 1317-1323.

[2] H. Bodlaender, "On the complexity of some coloring games," Graph Theoretical Concepts in Computer Science, (R. Möhring, ed.), vol. 484, Lecture notes in Computer Science, Springer-Verlag, (1991) 30-40. 
[3] L. Cai and X. Zhu, "Game chromatic index of k-degenerate graphs," Journal of Graph Theory, 36 (2001), no. 3, 144-155.

[4] C. Chou, W. Wang, and X. Zhu, "Relaxed game chromatic number of graphs," Discrete Mathematics, 262 (2003) no. 1-3, 89-98.

[5] L. Cowen, R. Cowen, and D. Woodall, "Defective colorings of graphs in surfaces: partitions into subgraphs of bounded valency," Journal of Graph Theory, 10 (1986), 187-195.

[6] L. Cowen, W. Goddard, and C. Jesurum, "Defective coloring revisited," Journal of Graph Theory, 24 (1997), 205-220.

[7] W. Deuber and X. Zhu, "Relaxed coloring of a graph," Graphs Combin., 14 (1998), $121-130$.

[8] T. Dinski and X. Zhu, "A bound for the game chromatic number of graphs," Discrete Mathematics, 196 (1999), 109-115.

[9] C. Dunn, "Complete multipartite graphs and the relaxed coloring game," Order, 29, Issue 3 (2012), 507-512.

[10] C. Dunn, "The relaxed game chromatic index of $k$-degenerate graphs," Discrete Mathematics, 307 (2007), 1767-1775.

[11] C. Dunn, H.A. Kierstead, "A simple competitive graph coloring algorithm II," Journal of Combinatorial Theory, Series B, 90 (2004) 93-106.

[12] C. Dunn, H.A. Kierstead, "A simple competitive graph coloring algorithm III," Journal of Combinatorial Theory, Series B, 92 (2004) 137-150.

[13] C. Dunn, H.A. Kierstead, "The relaxed game chromatic number of outerplanar graphs," Journal of Graph Theory, 46 (2004) 69-78.

[14] N. Eaton and T. Hull, "Defective list colorings of planar graphs," Bull. Inst. Combin. Appl., 25 (1999), 79-87.

[15] P. Erdös, U. Faigle, W. Hochstättler, W. Kern "Note on the game chromatic index of trees," Theoretical Computer Science, 313, Issue 3 (2004), 371-376.

[16] U. Faigle, W. Kern, H.A. Kierstead, and W. Trotter, "On the game chromatic number of some classes of graphs," Ars Combinatoria, 35 (1993), 143-150.

[17] M. Gardner, "Mathematical Games," Scientific American, (April, 1981) 23.

[18] D. Guan and X. Xhu, "Game chromatic number of outerplanar graphs," Journal of Graph Theory, 30 (1999), 67-70.

[19] W. He, J. Wu, and X. Zhu, "Relaxed game chromatic number of trees and outerplanar graphs," Discrete Mathematics, 281 (2004), no. 1-3, 209-219.

[20] H.A. Kierstead, "A simple competitive graph coloring algorithm," Journal of Combinatorial Theory, Series B, 78 (2000), 57-68.

[21] H.A. Kierstead and W. Trotter, "Planar graph coloring with an uncooperative partner," Journal of Graph Theory, 18 (1994), 569-584.

[22] H.A. Kierstead and Zs. Tuza, "Marking games and the oriented game chromatic number of partial k-trees," Graphs Combin., 19 (2003), no. 1, 121-129.

[23] P. Lam, W. Shiu, and B. Xu, "Edge game-coloring of graphs," Graph Theory Notes of New York XXXVII, (1999), 17-19.

[24] R. Škrekovski, List impoper colourings of planar graphs, Combin. Probab. Comput. 8 (1999), 293-299.

[25] X. Zhu, "Game coloring of planar graphs," Journal of Combinatorial Theory, Series $B, 75$ (1999), 245-258.

[26] X. Zhu, "The game coloring number of pseudo partial $k$-trees," Discrete Mathematics, 215 (2000), 245-262. 
Linfield College, McMinnville, OR 97128

E-mail address: cdunn@linfield.edu

University of Minnesota, Minneapolis, MN 55455

E-mail address: dmorawski@gmail.com

Linfield College, McMinnville, OR 97128

E-mail address: jfirkins@linfield.edu 